\title{
High Spatial Resolution Thermal Infrared Spectroscopy with ALES: Resolved Spectra of the Benchmark Brown Dwarf Binary HD 130948BC
}

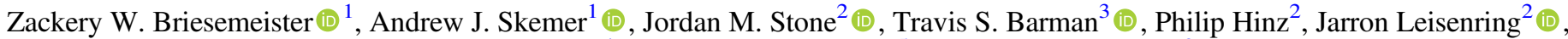 \\ Michael F. Skrutskie ${ }^{4}$, Charles E. Woodward ${ }^{5}$, and Eckhart Spalding ${ }^{2}$ (I) \\ ${ }^{1}$ Department of Astronomy and Astrophysics, University of California, 1156 High Street, Santa Cruz, CA 95064, USA; zbriesem@ucsc.edu \\ ${ }^{2}$ Steward Observatory, University of Arizona, 933 N. Cherry Avenue, Tucson, AZ 85719, USA \\ ${ }^{3}$ Lunar and Planetary Laboratory, University of Arizona, 1629 E University Boulevard, Tucson, AZ 85721-0092, USA \\ ${ }^{4}$ Department of Astronomy, University of Virginia, 530 McCormick Road, Charlottesville, VA 22904, USA \\ ${ }^{5}$ Minnesota Institute for Astrophysics, University of Minnesota, 116 Church Street, S.E. Minneapolis, MN 55455, USA \\ Received 2018 October 30; revised 2019 April 11; accepted 2019 April 12; published 2019 May 30
}

\begin{abstract}
We present 2.9-4.1 $\mu \mathrm{m}$ integral field spectroscopy of the L4+L4 brown dwarf binary HD 130948BC, obtained with the Arizona Lenslets for Exoplanet Spectroscopy (ALES) mode of the Large Binocular Telescope Interferometer. The HD 130948 system is a hierarchical triple system, in which the G2V primary is joined by two co-orbiting brown dwarfs. By combining the age of the system with the dynamical masses and luminosities of the substellar companions, we can test evolutionary models of cool brown dwarfs and extrasolar giant planets. Previous near-infrared studies suggest a disagreement between HD 130948BC luminosities and those derived from evolutionary models. We obtained spatially resolved, low-resolution $(R \sim 20) L$-band spectra of HD 130948B and $\mathrm{C}$ to extend the wavelength coverage into the thermal infrared. Jointly using $J H K$ photometry and ALES $L$-band spectra for HD 130948BC, we derive atmospheric parameters that are consistent with parameters derived from evolutionary models. We leverage the consistency of these atmospheric quantities to favor a younger age $(0.50 \pm 0.07 \mathrm{Gyr})$ of the system compared to the older age $\left(0.79_{-0.15}^{+0.22} \mathrm{Gyr}\right)$ determined with gyrochronology in order to address the luminosity discrepancy.
\end{abstract}

Key words: brown dwarfs - infrared: stars - techniques: imaging spectroscopy

\section{Introduction}

Near-infrared $(1-2.5 \mu \mathrm{m})$ adaptive-optics-fed integral field spectrographs (IFSs; OSIRIS, Larkin et al. 2006; GPI, Macintosh et al. 2008; SPHERE, Claudi et al. 2008; Project 1640, Hinkley et al. 2011; CHARIS, McElwain et al. 2012) have been detecting and characterizing exoplanets in high-contrast regimes for nearly a decade (e.g., Bowler et al. 2010; Barman et al. 2011). Because each wavelength slice of a data cube from an IFS can be analyzed using techniques for high-contrast image processing, IFSs are uniquely suited for high-contrast spectroscopy. Furthermore, planet-star spectral diversity can be harnessed to deliver better high-contrast imaging performance and sensitivity to planets compared to more traditional imagers (e.g., Zurlo et al. 2014).

While IFSs are uniquely capable of obtaining spatially resolved spectra of exoplanets, adaptive-optics-fed IFSs have been confined to the optical and near-infrared $(<3 \mu \mathrm{m})$. Nearinfrared spectra alone are insufficient for precise atmospheric constraints of brown dwarfs and exoplanets, due to degeneracies between effective temperature, cloud coverage, convection, and non-equilibrium carbon chemistry (e.g., Stephens et al. 2009; Skemer et al. 2014; Barman et al. 2015). Previous works have exploited broad wavelength spectrophotometry extending into the thermal infrared in order to constrain the thermal profiles, compositions, cloud properties, and bolometric luminosities of gas-giant planets (e.g., Barman et al. 2011; Currie et al. 2011; Madhusudhan et al. 2011; Marley et al. 2012; Skemer et al. 2012, 2014; Ingraham et al. 2014; Morzinski et al. 2015; Rajan et al. 2017).

In the thermal infrared $(3-5 \mu \mathrm{m})$, the spectral energy distribution (SED) of gas-giant planets contains a low-opacity atmospheric window that emits a large fraction of a planet's flux (Low \& Davidson 1969; Bjoraker et al. 1986; Burrows et al. 1997), especially at cool temperatures (see Figure 1). Major atmospheric absorbers, such as $\mathrm{CH}_{4}, \mathrm{CO}$, and $\mathrm{H}_{2} \mathrm{O}$, have strong absorption features at $\sim 3.3 \mu \mathrm{m}, \sim 4.7 \mu \mathrm{m}$, and $\sim 4-5 \mu \mathrm{m}$, respectively (Morley et al. 2014). In addition, the thermal infrared continuum shape is sensitive to cloud thickness and patchiness (Currie et al. 2011; Madhusudhan et al. 2011; Skemer et al. 2014).

We built the Arizona Lenslets for Exoplanet Spectroscopy (ALES; Skemer et al. 2015) to extend the spectroscopic wavelength coverage available for directly imaged planets in order to better understand their atmospheric processes. ALES is the world's first adaptive-optics-fed thermal infrared IFS and exists as a mode of LMIRcam (Skrutskie et al. 2010; Leisenring et al. 2012) - the 1-5 $\mu \mathrm{m}$ adaptive-optics (AO) imager for the Large Binocular Telescope Interferometer (LBTI; Hinz et al. 2008a, 2012, 2014). With ALES, we can exploit these tools developed for high-contrast imaging to probe longer wavelengths and cooler effective temperatures (Figure 1).

In this work, we present a commissioning data set for ALES: the HD 130948 hierarchical triple system comprising an L4 + L4 brown dwarf binary - separated by $\lesssim 110$ mas - on a wide orbit $(\sim 2$ !. 6$)$ around a Sun-like primary star $(\mathrm{G} 2 \mathrm{~V},[\mathrm{M} / \mathrm{H}]=$ 0.0; Potter et al. 2002). The HD 130948 system offers a rare laboratory to test substellar evolutionary models due to the independent measurements of age (from gyrochronology and chromospheric activity of the primary star), luminosity (from photometry and spectroscopy of the brown dwarfs themselves), and total mass of the brown dwarf pair (from orbital motion; Dupuy et al. 2009). While HD 130948B and C are distinct from exoplanets, tests of substellar evolutionary models are key 

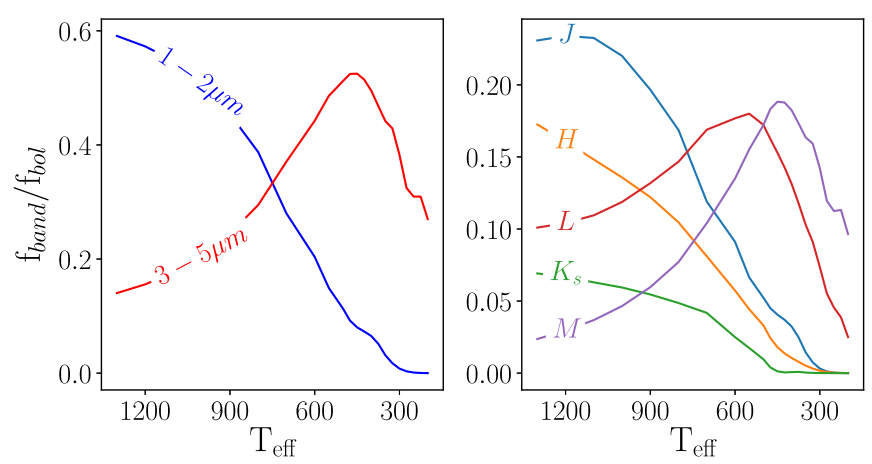

Figure 1. Left: thermal emission with respect to bolometric flux in 1-2 and 3-5 $\mu \mathrm{m}$ for cold exoplanets. Right: thermal emission with respect to bolometric flux in each band for cold exoplanets. The $1-2 \mu \mathrm{m}$ contribution to the bolometric flux is negligible for the coldest objects. Models from Morley et al. (2012, 2014).

to calibrating models and improving our ability to understand exoplanet observations. Previous comparisons with evolutionary models suggest HD 130948B and HD 130948C are two to three times more luminous than predicted (Dupuy et al. 2009, 2014; Dupuy \& Liu 2017). In this work, we extend the spatially resolved flux constraints to longer wavelengths, providing a $2.9-4.1 \mu \mathrm{m}$ spectrum of each component.

In Sections 2 and 3, we present our ALES observations of HD 130948 along with our reductions. We extract and calibrate spatially resolved spectra in Section 4. We fit custom model atmospheres to our spectra and perform evolutionary model fitting with the derived bolometric luminosities and dynamical mass constraints to determine their individual masses in Section 5. In Section 6, we discuss the implications of our results. We derive bolometric luminosities for each brown dwarf using a combination of near-infrared photometry and ALES L-band spectroscopy. The measured bolometric luminosity for each source is brighter than predicted by evolutionary models given the gyro-age of the primary star. We also check the consistency of the evolutionary models with the atmosphere parameters derived in the spectral fitting. We summarize our conclusions in Section 7.

\section{Observations}

We observed HD 130948 on 2016 March 26-28 UT as part of early commissioning activities with ALES. We used the left (SX) primary mirror of the two $8.4 \mathrm{~m}$ primary mirrors of the LBT during photometric conditions and subarcsecond seeing. Visible light is directed toward the LBTI wavefront sensors for AO correction (Bailey et al. 2014) performed with the deformable secondary mirror (Esposito et al. 2011). Wavefront-corrected, diffraction-limited, infrared light is directed into the cryogenic Universal Beam Combiner and then into the Nulling and Imaging Camera (Hinz et al. 2008b) where LMIRcam (Skrutskie et al. 2010; Leisenring et al. 2012) resides.

For ALES operations, an $8 \times$ Keplerian magnifier, a silicon lenslet array with a pinhole grid to suppress diffraction, a blocking filter, and disperser (direct-vision prism assembly) are introduced into the light path via LMIRcam filter wheels. The light incident on the spatial extent of each lenslet is focused through the diffraction-suppressing pinhole grid. Each lenslet sub-image is then dispersed by the direct-vision prism assembly. These dispersed sub-images are imaged onto a
Table 1

HD 130948 Observations for 2016 March 28

\begin{tabular}{lcccr}
\hline \hline Observation & $N_{\text {pointings }}$ & $N_{\text {frames }}$ & $N_{\text {coadds }}$ & DIT $^{\text {a }}$ \\
\hline HD 130948A & 24 & 5 & 2 & $1 \mathrm{~s}$ \\
HD 130948BC & 24 & 30 & 2 & $1 \mathrm{~s}$ \\
Sky & 24 & 30 & 2 & $1 \mathrm{~s}$ \\
Darks & 24 & 9 & 1 & $1 \mathrm{~s}$
\end{tabular}

Note.

${ }^{\mathrm{a}}$ Detector integration time.

$5.2 \mu \mathrm{m}$ cutoff Teledyne HAWAII-2RG (H2RG, Beletic et al. 2008) as a grid of thermal infrared spectra.

At the time of observation, the FORCAST readout electronics (Leisenring et al. 2010) limited the detector readout to $1024 \times 1024$ pixels, instead of the complete $2048 \times 2048$ pixels of the H2RG. This subarray contains $50 \times 50 \mathrm{~L}$-band spectra with spectral resolution $R \sim 20$, covering a field of view of $1 . " 3 \times 1$."3. Skemer et al. (2015) presented a description of the design of the version of ALES used in this paper. Subsequent upgrades to ALES, available for current and future use, are described by Hinz et al. (2018) and Skemer et al. (2018).

For this data set, we took natural guide star AO observations of the hierarchical triple system HD 130948, using HD 130948A as the reference star. The spatial scale of the HD 130948 system is larger than the field of view of ALES (the binary is separated by 2". 6 from the primary). Therefore, the data were obtained in a three-point pattern consisting of HD 130948A, sky, and HD 130948BC. Dark frames were obtained between each nod position while the telescope was in motion. A detailed description of this strategy can be found in Stone et al. (2018). Overall, we obtained the data described in Table 1. Four of the twenty-four pointings were discarded: two had the binary positioned close to the edge of the lenslet array and two had poor AO correction.

Wavelength calibration of the low-resolution spectra is performed using dome flats at four spectrally unresolved narrowband ( $R \sim 100$ ) filters spanning 2.9-3.9 $\mu \mathrm{m}$ (Stone et al. 2018). An empirical dispersion relation is used to propagate the wavelength solution between the four data points for each spectrum.

\section{Reduction}

A raw ALES L-band frame consists of a grid of 2500 spectra, each spanning $\sim 37 \times 7$ pixels, dispersed in a grid at a fiducial angle $\theta=\tan ^{-1}\left(\frac{1}{2}\right)$ with respect to detector rows and columns. Raw ALES data were reduced using the ALES Python pipeline (MEAD; Briesemeister et al. 2018), with the steps performed as follows.

The first step performed by the pipeline is the removal of detector artifacts, including the removal of residual detector channel bias, pixel flat-fielding, background subtraction, and bad pixel correction.

For nonlinearity correction, we constructed fluence-to-count curves for each pixel by taking sky flats of varying integration times without ALES optics in the light path. The sky flats were bias-subtracted and flat-fielded. Outlier pixels were flagged as bad pixels. The linearity correction was applied to all frames. Frames of HD 130948BC had flux corrections $<0.01 \%$, sky frames had 
flux corrections of $<0.5 \%$, and frames of HD 130948A had flux corrections of $\sim 2 \%$.

The $3.9 \mu \mathrm{m}$ narrowband filter calibration data are used to coarsely locate the spectra on the detector. However, the calibration data for observations of HD 130948 on 2016 March 28 were taken at the end of the night; the telescope was set to zenith and the telescope experienced a different gravity vector. The irreproducibility of the multiple filter wheel positions, flexure, and the use of a distinct pupil stop for the calibration data resulted in a field-dependent $(\lesssim 1$ pixel $)$ shift between the calibration data and the science data. The deviations of the calibration data from science data, as well as the deviation of the dispersion direction from the fiducial angle, are calculated for each spaxel to parameterize a mapping of every light-sensitive pixel to a wavelength-calibrated $(\lambda, y, x)$ data cube. In order to turn the raw data into wavelength-calibrated data cubes, we apply optimal extraction (Horne 1986) to each spectrum, which becomes the associated spaxel in the data cube.

In a lenslet-based IFS, each lenslet has a slightly different throughput as a function of wavelength. To address this effect, a lenslet flat field is constructed from the normalized, darksubtracted sky data cube. This is necessary because, by design, LBTI runs in pupil-tracking mode only and does not rotate with the sky; astrophysical light of a binary system is incident on different lenslets over the course of observation, due to sky rotation.

The cubes containing HD 130948BC and the cubes containing HD 130948A were derotated by the median parallactic angle during each HD 130948BC pointing. Each wavelength slice of every cube was registered with respect to the appropriate wavelength slice of the highest signal-to-noise data cube using the single-step Discrete Fourier Transform (DFT) approach outlined in Guizar-Sicairos et al. (2008). This registration technique calculates pixel-accuracy translations using DFT phase correlation and then upsamples the crosscorrelation by a factor of 1000 in a $1.5 \times 1.5$ pixel neighborhood of the estimated pixel shift for subpixel-accuracy translation.

Once the translations of the derotated cubes are determined, the registered, derotated cubes are made with a final interpolation of the original cubes that performs the derotation and registration simultaneously in order to avoid multiple interpolations. The sum of the science and sky variance images undergoes the same optimal extraction process to be turned into data cubes, and the respective registration and derotation in a single step. The average of the group of data cubes, weighted by their respective inverse variance cubes, become the final $(\lambda, y, x)$ data cubes of HD 130948BC and HD 130948A. The final $(\lambda, y, x)$ variance cubes are the mean of all propagated variance cubes. These final cubes and associated propagated variance cubes are then used for point-spread function (PSF) photometry.

The final cube for HD 130948BC is shown in Figure 2, in which the binary is centered in each wavelength frame denoted by their respective wavelengths. The $\sim 110$ mas binary is resolved in the $L$-band by ALES. Each image is $50 \times$ 50 pixels, with one pixel corresponding to 26.1 mas. The binary appears brightest in the middle of the band because the sky transmission of the $L$-band peaks at $\sim 3.6 \mu \mathrm{m}$. The noise appears worse near the edges of each frame because fewer data cubes overlap in these regions.

\section{PSF Photometry}

We used PSF fitting to measure the flux from each component at each wavelength slice, explicitly allowing for spatial covariance in the images (see the appendix for more details). We took a two-step approach, first fitting for binary separation and position angle, and then extracting the flux with the respective priors of those two parameters. A two-step process is required because the position angle $\theta$ and separation $\rho$ of the two brown dwarfs are expected to be constant across all wavelengths, while the position of the binary in the data cube can shift as a function of wavelength due to chromatic optics and atmospheric dispersion. The first step performs PSF subtraction at each wavelength slice completely independently. The second step repeats the PSF fitting at each wavelength slice, including Gaussian priors for the position angle $\theta$ and separation $\rho$, with hyperparameters derived from the first step.

Due to the AO correction, the HD 130948BC pair is far enough away from the primary star such that high-contrast postprocessing algorithms for PSF subtraction are not used, so PSF subtraction does not dominate the correlation of noise in our data. However, interpolation and diffraction still induce spatial and spectral correlations for the HD 130948BC data set. We extended the Bayesian framework used in Wang et al. (2016), which coupled Bayesian parameter estimation for astrometry with a Gaussian process, to apply to a binary system in order to account for the spatial noise covariance caused by interpolation during data reduction and the formation of data cubes. Because the squared exponential covariance function would produce improbably smooth noise realizations, we parameterized the spatial noise covariance by the Matern $(\nu=3 / 2)$ covariance function

$$
C_{\ell, i j}=\sigma_{i} \sigma_{j}\left(1+\frac{\sqrt{3} r_{i j}}{\ell}\right) \exp \left(\frac{-\sqrt{3} r_{i j}}{\ell}\right),
$$

where $\ell$ is the spatial correlation length of noise, $r_{i j}$ is the Euclidean distance between $i, j$ spaxels, and $\sigma_{i}$ is the standard deviation associated with spaxel $i$. The spatial correlation length $\ell$ represents the strength of correlation between two spaxels averaged across the entire wavelength slice.

We remain agnostic to spectral correlation when each wavelength slice is treated independently from one another. However, imposing Gaussian priors on $\theta$ and $\rho$ during the second step does introduce correlation between all wavelength slices. Interpolation and finite spectral resolution also contribute to spectral correlation. The characterization and treatment of spectral correlation is discussed in Section 5.1.

The details of the PSF-fitting procedure are available in the appendix. We evaluated convergence with acceptance rates and the multivariate Gelman-Rubin convergence diagnostic (Gelman \& Rubin 1992; Brooks \& Gelman 1998). The best-fit model at each wavelength was identified as the median of the marginalized posteriors, with $68 \%$ credible regions of the marginalized posteriors as their uncertainty. Representative best-fit models and residuals at the $3.03,3.47$, and $3.87 \mu \mathrm{m}$ wavelength slices of the data cube are shown in Figure 3, and the marginalized posteriors following the second PSF-fitting step for the $3.47 \mu \mathrm{m}$ wavelength slice are shown in Figure 4 . The medians of the marginalized posterior distributions from the second step remained within the corresponding $68 \%$ credible regions of the marginalized posteriors from the first 


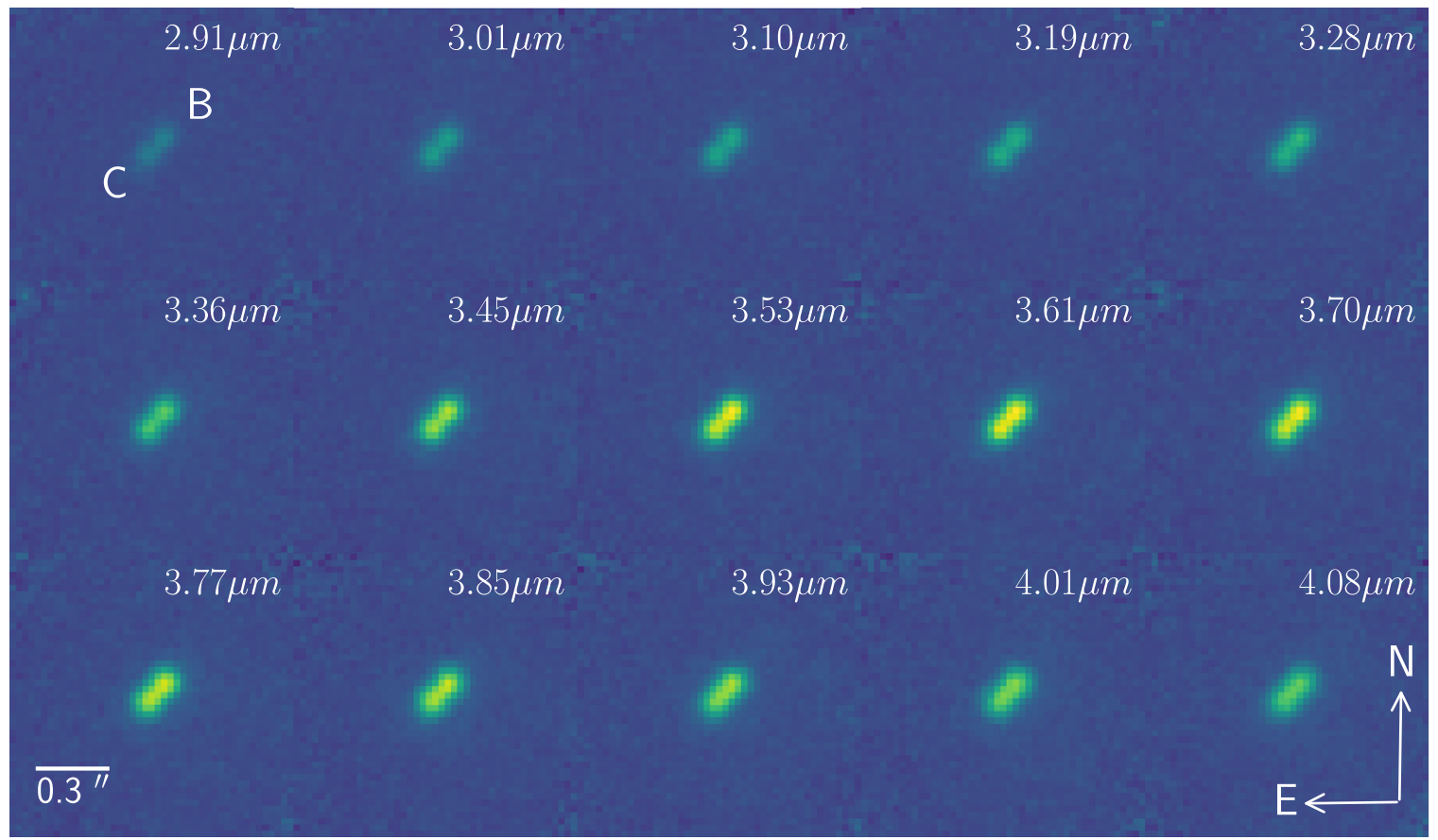

Figure 2. ALES data cube of HD 130948BC, where each image is a slice of the cube at the wavelengths specified at the upper-right corner. These are 15 representative wavelength slices of the 35 wavelength slices in each data cube.
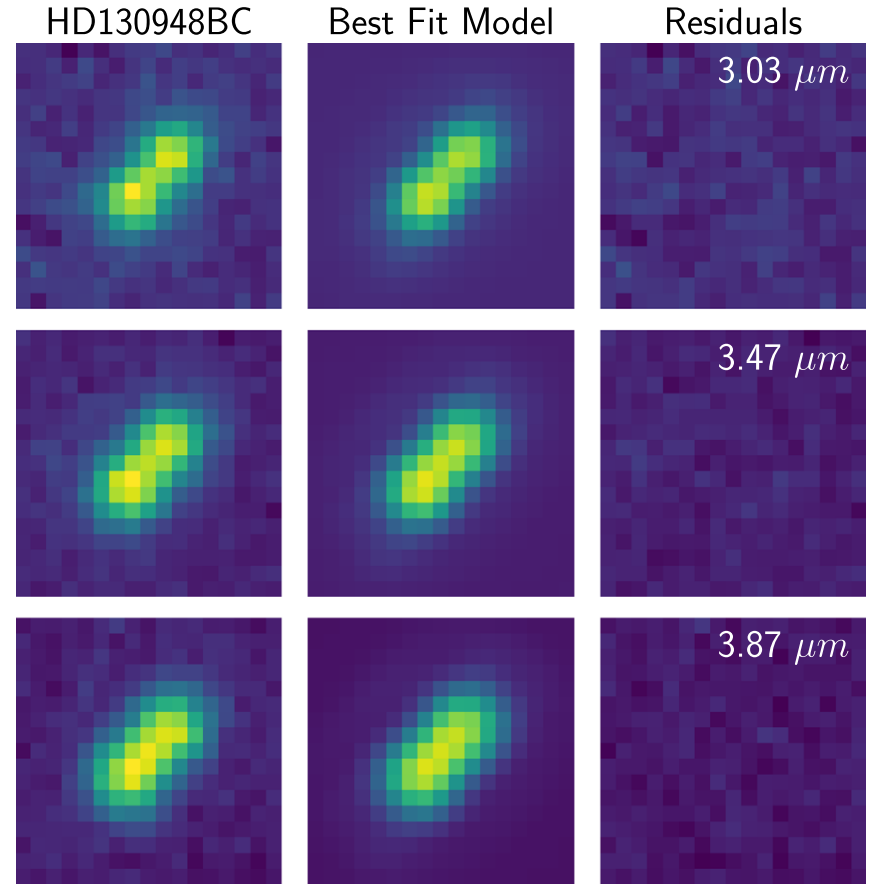

Figure 3. The first image in each row is a representative data cube slice of HD $130948 \mathrm{BC}$ at the wavelength denoted in the upper-right corner of the row. The middle image in each row is the best-fit model of two scaled PSFs that fit the binary. The right image depicts their residuals. Each wavelength of the cube produced quantitatively similar results. The color stretch is linear.

step, with the exception of the far red end $(>4 \mu \mathrm{m})$ of the band, where the PSF is larger and the binary is less resolved.

The spatial correlation lengths $\ell(\lambda)$ were determined empirically to trend linearly with wavelength with the form $\ell(\lambda)=(0.115 \pm$ $\left.0.008 \mathrm{px} \mu \mathrm{m}^{-1}\right) \lambda+(0.236 \pm 0.006 \mathrm{px})$. The amplitude of the spatial correlation is not consistent with the diffraction limit of the telescope ( $\sim 4 \mathrm{px})$, suggesting that interpolation from data cube construction and PSF fitting is contributing to small-scale correlation, the ALES data cubes of HD 130948BC are not speckle limited, and the mean photon noise is background dominated. The PSF-fitting procedure was repeated for data cubes and registration performed by strictly linear, cubic, and quintic interpolation, and permutations thereof, and the spatial correlation lengths remained between 0.5 and 0.9 px. The Bayesian parameter estimation of the other model parameters were unchanged with the different interpolation schemes.

The contrast spectrum for each brown dwarf is derived with respect to the primary star HD 130948, a G2V star with solar metallicity (Valenti \& Fischer 2005). A model G2V spectrum (Hauschildt et al. 1999) was smoothed to the spectral resolution of ALES and scaled by the WISE WI $(3.3526 \mu \mathrm{m}$; Wright et al. 2010) photometry data point of HD 130948 A of $5.17 \pm 0.40 \mathrm{Jy}$ (Eiroa et al. 2013). HD 130948BC do not contribute significantly to the WISE W1 photometry of HD 130948A, as they are $7 \mathrm{mag}$ dimmer. The spatially resolved contrast spectra of HD 130948B and C were then multiplied by the scaled model to get the absolute flux-calibrated spectra for HD 130948BC with uncertainties propagated.

\section{Analysis}

\subsection{Physical Parameters from Atmospheric Models}

With the goal of providing a consistent description of the atmospheres of HD $130948 \mathrm{BC}$ using $J H K$ photometry and $L$ band spectra, we will explore atmospheric model fitting of solely $L$-band spectra, solely NIR photometry, and joint NIR photometry plus $L$-band spectra.

The PHOENIX atmospheric code outlined in Barman et al. (2011) was used to calculate the synthetic model spectra for this analysis. We chose to interpolate over a presynthesized library 


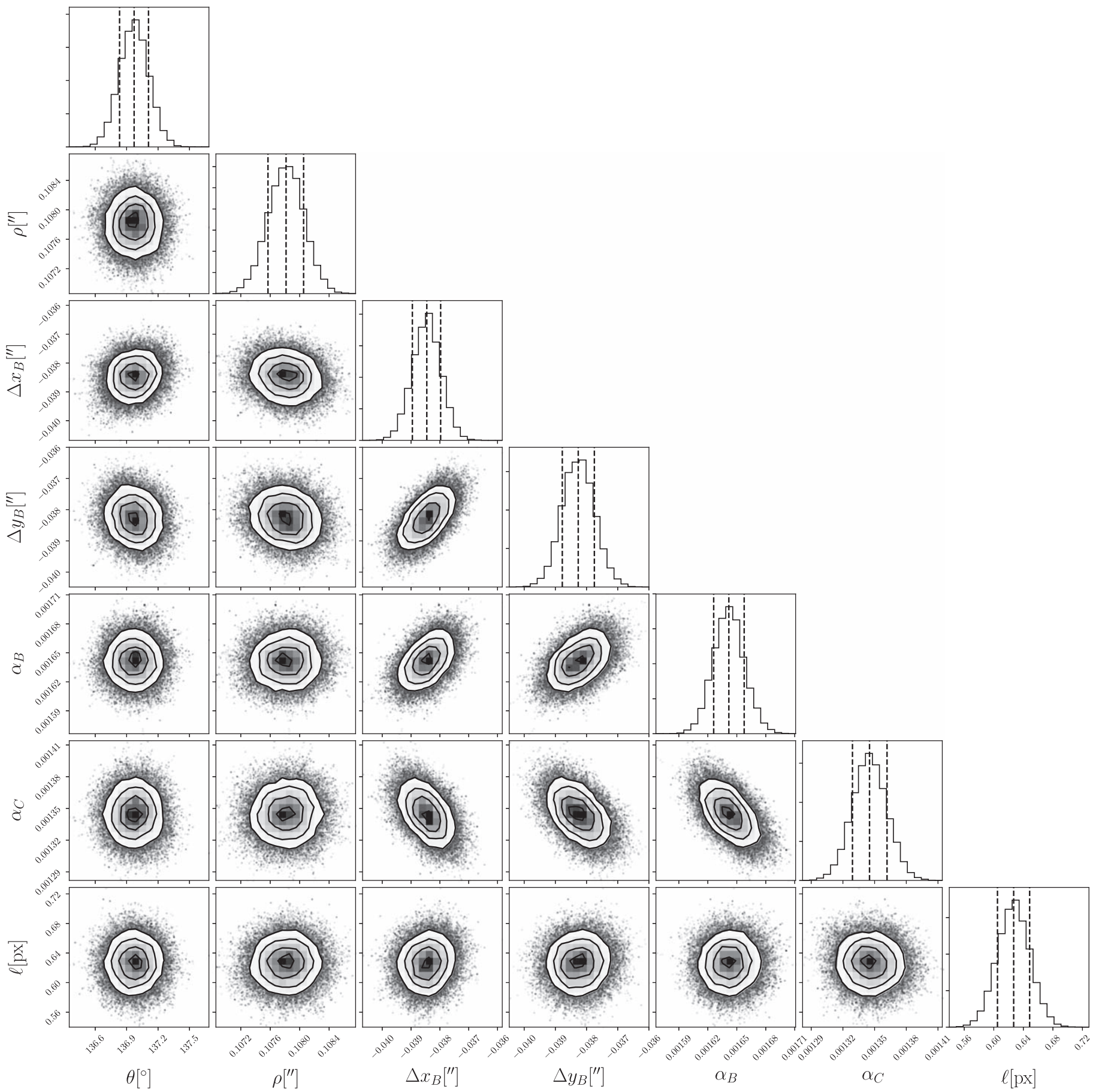

Figure 4. Posterior distributions for the position of HD 130948B and flux ratios of HD 130948BC with respect to HD 130948A in the representative 3.47 $\mu \mathrm{m}$ wavelength slice. The posteriors are calculated at each wavelength, and the flux ratios are used to derive the spectrum of HD 130948BC by multiplying with the spectrum of HD 130948A. The contour levels are set to intervals of $0.5 \sigma$.

of model spectra calculated between 1500 and $2500 \mathrm{~K}$ and $\log$ $g=4.5$ and $\log g=5.5$, with resolutions of $\Delta T_{\mathrm{eff}}=100 \mathrm{~K}$, $\Delta \log g=0.5$ dex. The grid of spectra were log-linear interpolated at the native resolution of the spectra to obtain the flux density for arbitrary $k=\left[T_{\text {eff }}, \log g\right]$. More sophisticated methods of spectral interpolation, such as Starfish (Czekala et al. 2015), were not used because the spectral resolution of ALES cubes requires convolution and downsampling that washes out the noding phenomenon described therein.

\subsubsection{Modeling of ALES L-band Spectra}

Most IFS data sets are prone to spectral and spatial correlation. The ALES HD 130948 data set is no different. In Section 4, we showed that there is a nonnegligible spatial correlation on subpixel scale. In Greco \& Brandt (2016), a procedure for modeling spectral correlation is outlined, in which a three-component model is proposed to characterize contributions of speckle noise, correlation induced by interpolation during reduction, and uncorrelated noise. With the formalism from Greco \& Brandt, we estimated the 

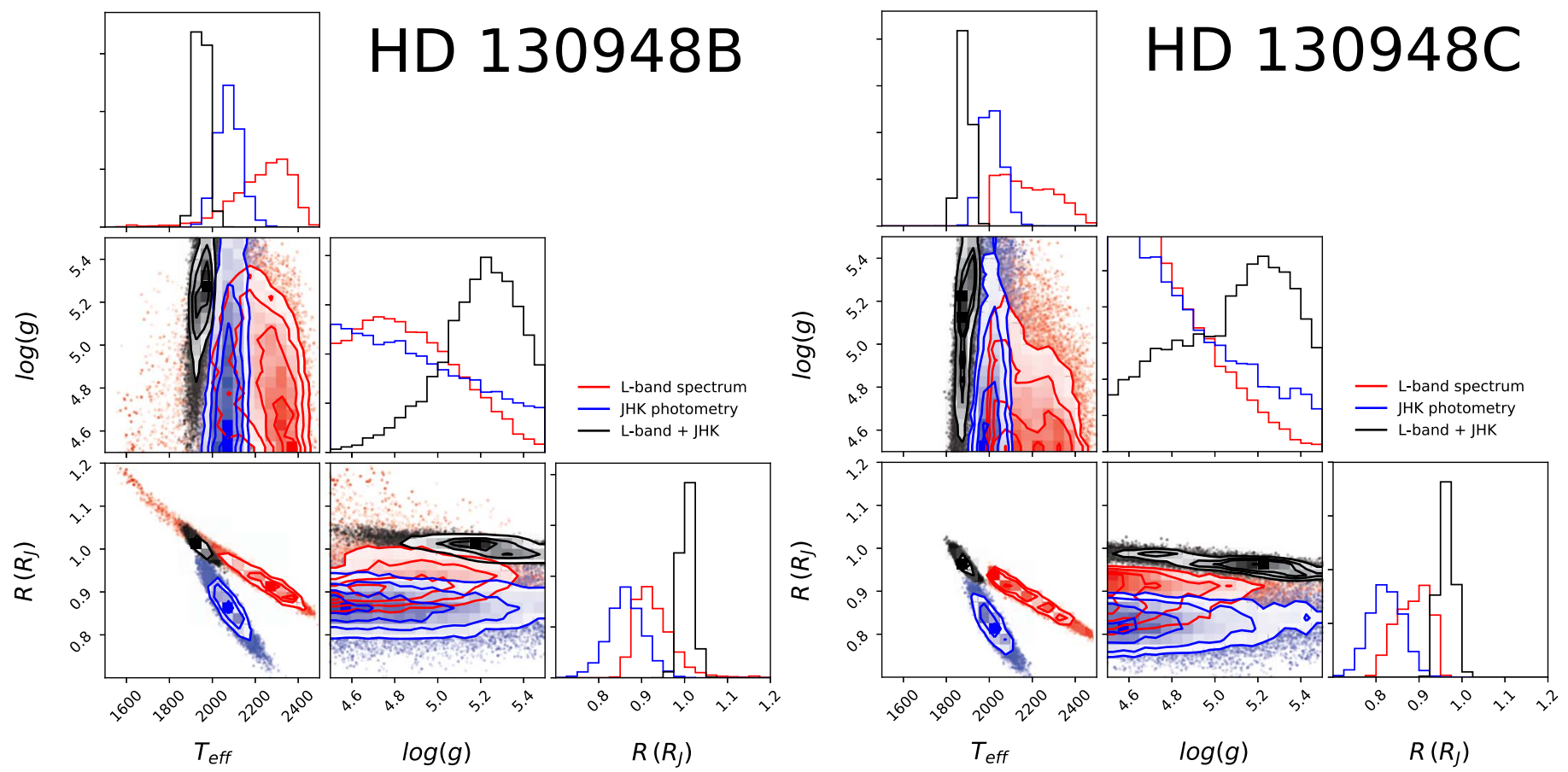

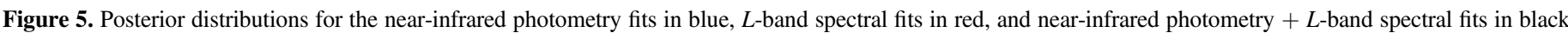

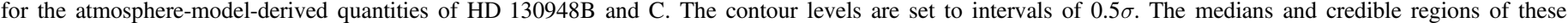

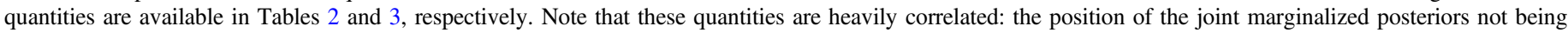

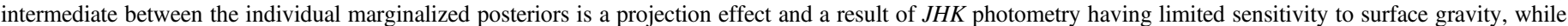
$L$-band spectra have limited sensitivity to temperature for hot substellar atmospheres.

correlation $\psi_{i j}$ between pixel values at wavelengths $\lambda_{i}$ and $\lambda_{j}$ within an annulus of width $1.5 \lambda_{c} / D$ in the binary data with the binary masked out $\left(\lambda_{c}=3.50 \mu \mathrm{m}\right)$. The correlation was fit with a three-component model, comprising an uncorrelated term with amplitude $A_{\delta}$, spatially independent Gaussian term with amplitude $A_{\lambda}$ and correlation length $\sigma_{\lambda}$, and a spatially dependent Gaussian term with amplitude $A_{\rho}$ and correlation length $\sigma_{\rho}$. The respective amplitudes were fit such that $A_{\delta}=0.55, A_{\lambda}=0.27$, and $A_{\rho}=$ 0.18 . The noise components were characterized by correlation lengths $\sigma_{\rho}=0.81$ and $\sigma_{\lambda}=0.015$. The spectra are correlated to $\sim 2$ channels, supporting our assumption that we have $N_{\lambda} / 2$ resolution elements for critical Nyquist sampling in the appendix. This method is not sensitive to the subpixel spatial correlation measured in Section 4 because there are no appropriate annuli to measure these lengths.

Bayes' theorem is used to write the posterior probability for $k=\left[T_{\text {eff }}, \log (g)\right]$ given an observed spectrum $f$ as $\mathcal{P}(k \mid f) \propto$ $\mathcal{L}(f \mid k) \mathcal{P}(k)$. To quantify the probability of the data conditioned on the model $\mathcal{L}(f \mid k)$, we adopted the following multivariate Gaussian likelihood function (ignoring constants):

$$
-2 \ln \mathcal{L}(f \mid k)=\left(f-\alpha F_{k}\right)^{T} \Sigma^{-1}\left(f-\alpha F_{k}\right),
$$

where $F_{k}$ is the synthetic spectrum for model $k, \Sigma_{i j}=\sigma_{i} \sigma_{j} \psi_{i j}$ for flux errors $\sigma$, and $\alpha=(R / D)^{2}$ for brown dwarf radius $R$ and distance $D$ to the system.

The prior on the surface gravity and temperature is defined by the domain limits: $\mathcal{P}(\log (g)[\operatorname{cgs}])=\mathcal{U}[4.5,5.5]$ and $\mathcal{P}\left(T_{\text {eff }}[\mathrm{K}]\right)=\mathcal{U}[1500,2500]$. Realistic priors from evolutionary models could be applicable here. However, Dupuy et al. (2010) discussed the existence of a systematic discrepancy between atmosphere and evolutionary-model-derived quantities and evolutionary-model-predicted quantities from data. It is for this reason that we avoid using the evolutionary models as realistic priors in the atmospheric model fitting suggested in Greco \& Brandt (2016).

Gaia DR2 provides revised parallax measurements of HD 130948A of $\pi=54.91 \pm .07$ mas (Gaia Collaboration et al. 2016, 2018). The presence of brown dwarf companions will cause deviations from parallactic trajectory with linear proper motion due to stellar reflex motion, but the lower bound on the orbital period is $155 \mathrm{yr}$ (Ginski et al. 2013); only a fraction of the orbit is traversed during the Gaia baseline, suppressing the reflex motion signal. Moreover, HD 130948 is a bright star $(G=5.715)$ in the Gaia catalog, in which stars with $G \lesssim 6$ have weaker positional accuracy due to saturation of the detector, placing reflex motion signal below the noise floor associated with the parallax uncertainty. We proposed Gaussian priors for parallax $\pi$ to estimate the bounded prior on distance as follows: $\mathcal{P}(D[\mathrm{pc}])=$ $\left|\frac{\partial \pi}{\partial D}\right| \cdot \mathcal{N}\left[\pi ; \pi, \sigma_{\pi}^{2}\right]=D^{-2} \mathcal{N}\left[1 / D ; \pi, \sigma_{\pi}^{2}\right]$. The prior for brown dwarf radius was set to $\mathcal{P}\left(R\left[R_{\mathrm{J}}\right]\right)=\mathcal{L U}[0.5,1.5]$, with physically motivated limits from evolutionary models and a lack of high insolation.

We used the Goodman \& Weare (2010) affine-invariant MCMC sampler implemented in the emcee Python package (Foreman-Mackey et al. 2013) to sample the posterior distribution of $\left[T_{\text {eff }}, \log g, R, D\right]$ for HD 130948B and HD $130948 \mathrm{C}$ independently. We initialized 50 walkers with a guess $k$-vector plus Gaussian noise at an amplitude of $k \times 10^{-4}$. Each MCMC sampler was run for 400 steps after 400 burn-in steps. We evaluated the convergence of the chains with the Gelman-Rubin convergence diagnostic. The resulting marginalized posteriors can be seen in red in Figure 5. The median and credible regions of these posteriors are reported in Tables 2 and 3. The best-fit $L$-band spectra of HD 130948B 
Table 2

Atmosphere-model-inferred Properties of HD 130948B

\begin{tabular}{lccc}
\hline \hline Property & $J H K$ & $L$ & $J H K+L$ \\
\hline$T_{\text {eff }}[\mathrm{K}]$ & $2060 \pm 60$ & $2260_{-150}^{+100}$ & $1950 \pm 30$ \\
Radius $\left[R_{\mathrm{J}}\right]$ & $0.87 \pm 0.04$ & $0.92_{-0.03}^{+0.04}$ & $1.00 \pm 0.01$ \\
$\log (g)\left[\mathrm{cm} \mathrm{s}^{-2}\right]$ & $4.5_{-0.5}^{+0.7}$ & $4.7_{-0.4}^{+0.3}$ & $5.2 \pm 0.3$ \\
$\log \left(L_{\text {bol }}\right)\left[L_{\odot}\right]$ & $-3.88 \pm 0.02$ & $-3.87 \pm 0.02$ & $-3.87 \pm 0.01$ \\
\hline
\end{tabular}

Table 3

Atmosphere-model-inferred Properties of HD 130948C

\begin{tabular}{lccc}
\hline \hline Property & $J H K$ & $L$ & $J H K+L$ \\
\hline$T_{\text {eff }}[\mathrm{K}]$ & $2000 \pm 60$ & $2200_{-180}^{+150}$ & $1870 \pm 30$ \\
Radius $\left[R_{\mathrm{J}}\right]$ & $0.83 \pm 0.04$ & $0.87_{-0.03}^{+0.06}$ & $0.98 \pm 0.02$ \\
$\log (g)\left[\mathrm{cm} \mathrm{s}^{-2}\right]$ & $4.4_{-0.4}^{+0.5}$ & $4.3_{-0.3}^{+0.4}$ & $5.1 \pm 0.3$ \\
$\log \left(L_{\text {bol }}\right)\left[L_{\odot}\right]$ & $-3.97 \pm 0.02$ & $-3.97 \pm 0.02$ & $-3.96 \pm 0.01$ \\
\hline
\end{tabular}

and HD 130948C have $\chi_{\mathrm{B}}^{2}=13.7$ and $\chi_{\mathrm{C}}^{2}=21.1$. For 31 spectral bins and free parameters of $k=\left[T_{\mathrm{eff}}, \log g, R\right]$, these appear to be anomalously low $\chi^{2}$ values. However, the spectral correlation length of $\sim 2$ channels reduces the effective number of degrees of freedom by roughly half.

\subsubsection{Modeling of Near-infrared Photometry}

Dupuy et al. (2009) measured the MKO JHK photometry of HD 130948BC, which was updated with progressively homogenized analyses in Dupuy et al. (2014) and Dupuy \& Liu (2017). Crossfield (2014) presented 2MASS $J H K_{s}$ photometry that is consistent with the MKO photometry for both brown dwarfs. We chose to use the MKO photometry due to the smaller uncertainty in the photometry. We did not include any covariance in the $J H K$ photometry.

We approach atmospheric modeling of near-infrared photometry using $\chi^{2}$-fitting. Synthetic photometry $F_{k}$ was calculated for each model $k$ using MKO filter curves (Tokunaga et al. 2002). We used identical priors from Section 5.1.1, with the same motivations. To quantify the probability of the data conditioned on the model, we used Equation (2) in the regime where $\Sigma$ is diagonal. We sampled the posterior distribution of $\left[T_{\text {eff }}, \log g, R, D\right]$ for HD 130948B and HD 130948C independently with emcee under the same conditions as Section 5.1.1, satisfying the same convergence testing, and the resulting marginalized posteriors can be seen in blue in Figure 5. Median and credible regions of these posteriors are reported in Tables 2 and 3. The best-fit $J H K$ photometry of HD 130948B and HD 130948C have $\chi_{\mathrm{B}}^{2}=6.4$ and $\chi_{\mathrm{C}}^{2}=10.6$.

\subsubsection{Modeling of Photometry and Spectra}

Combining photometry and spectroscopy to derive meaningful atmospheric properties can unintentionally be driven by weighting schemes. The goodness-of-fit statistic $G_{k}$ from Cushing et al. (2008) is a commonly used statistic with weights proportional to the wavelength interval associated with the data points. While $G_{k}$ allows for heteroskedasticity, it implicitly assumes no correlation between spectral channels.

We approached this problem with two methods: apply no objective weighting scheme and use the covariance measured in the $L$-band, or apply objective weights according to Cushing et al. (2008) and ignore the covariance in the $L$-band. We chose to avoid extending the definition of the goodness-of-fit statistic $G_{k}$ to include covariance because it is not obvious whether the weighting scheme applies to off-diagonal covariance terms when the correlation $\psi_{i j}$ is measured in this manner.

We determined empirically that the posterior derived when applying no objective weights and using the covariance matrix completely contained the posterior when applying objective weights and ignoring the covariance. Therefore, we chose to ignore weights and favor including the covariance matrix. In principle, this means that near-infrared photometry is contributing less to the likelihood function.

We used identical priors from Section 5.1.1, with the same motivations. We also used the same functional form of the likelihood (Equation (2)), with $\psi_{i \neq j}=0$ for the three photometry points. We sampled the posterior distribution of $\left[T_{\text {eff }}, \log g, R, D\right]$ for HD 130948B and HD 130948C independently with emcee under the same conditions as Section 5.1.1, satisfying the same convergence testing, and the resulting marginalized posteriors can be seen in black in Figure 5. The median and credible regions of these posteriors are reported in Tables 2 and 3. The best-fit $J H K$ photometry and $L$-band spectra of HD 130948B and HD 130948C have $\chi_{\mathrm{B}}^{2}=20.1$ and $\chi_{\mathrm{C}}^{2}=31.8$.

Composite SEDs of $\mathrm{B}$ and $\mathrm{C}$ were built using $J H K$ photometry data over their respective bandpasses, the $L$-band spectra of B and C, and filling in the rest of the SED with the best-fit model spectra for each suite of fitting procedures (e.g., Morzinski et al. 2015). The bolometric luminosities were calculated by integrating these composite spectra. The uncertainty in the bolometric luminosities is derived using a Monte Carlo simulation, taking composite spectra drawn from both a multivariate Gaussian with the mean set to the spectrophotometry and covariance set to $\Sigma$ and model spectra from the respective posteriors, and estimating the standard deviation of their bolometric luminosities. We also performed a Monte Carlo simulation with SEDs associated with the best-fit model spectra of $\mathrm{B}$ and $\mathrm{C}$, excluding $J H K L$ data. The median remained unchanged, and the composite spectra method resulted in a larger uncertainty. We report the composite SED bolometric luminosity uncertainty in Tables 2 and 3.

Figure 6 contains the ALES L-band spectra of HD 130948B and $\mathrm{C}$, along with the model fits with parameters set to the medians of the marginalized posteriors from spectral fits for $J H K+L$ spectrophotometry. All of the following analyses are performed using these median and credible regions, reported in column $J H K+L$ in Tables 2 and 3.

\subsection{Individual Masses of HD $130948 B$ and $C$}

Benchmark systems like HD 130948BC provide the rare laboratory necessary to obtain individual masses of brown dwarfs, a measurement that is crucial to tests of evolutionary models. For most directly imaged planets, we must rely on evolutionary models to convert luminosity to mass given an age estimate (and potentially information on formation and initial entropy), so putting such models to the test is essential. In order to do this test, we need to isolate the mass of each object using the total mass constraint from orbital monitoring and our measurements of bolometric luminosity.

Substellar objects will tend to radiatively cool with time (Stevenson 1991; Burrows \& Liebert 1993); evolutionary models of substellar objects propose a luminosity-age-mass relationship, owing to a lack of a sustainable source of internal 


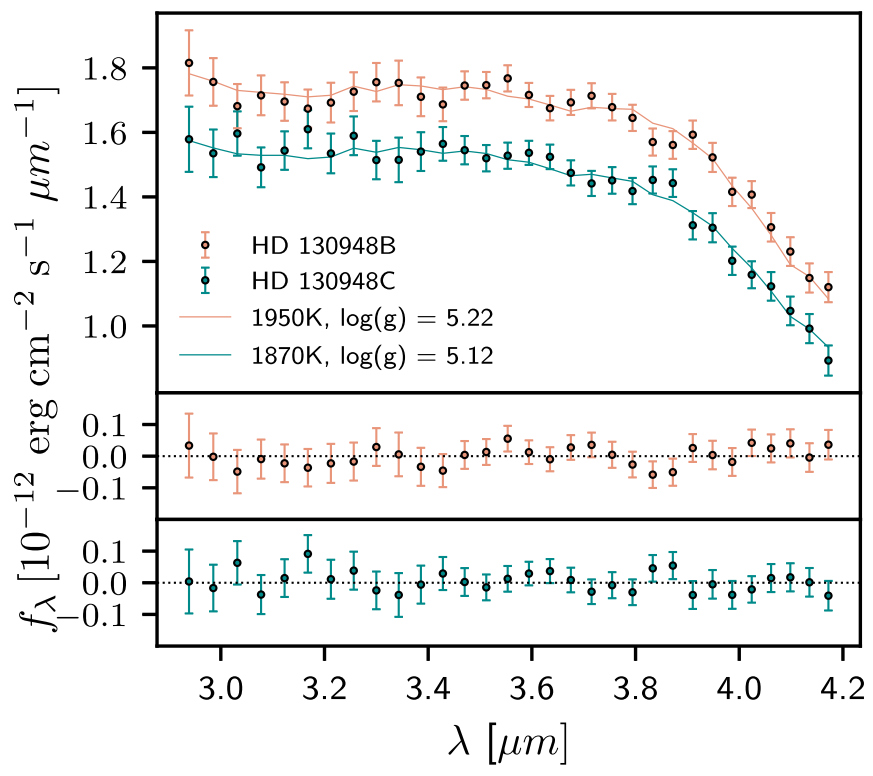

Figure 6. Spectra of HD 130948BC with best-fit models from Barman et al. (2011) using the near-infrared plus $L$-band fit. The residuals for each fit are plotted below with the color corresponding to the same object in the spectrum.

energy from nuclear reactions, with more massive objects starting hotter and more luminous. We exploit this relationship with evolutionary models from Baraffe et al. (2015) and Saumon \& Marley (2008) to isolate the masses and age of HD 130948BC. Because the measured luminosities are nearly equal, extreme mass ratios can be ruled out. Therefore, breaking the mass degeneracy is primarily driven by the tight dynamical mass and bolometric luminosity constraints instead of the evolutionary models themselves.

For individual ages between $1 \mathrm{Myr}$ and $1 \mathrm{Gyr}$, we calculate two masses with the prior that their sum is distributed according to the dynamical mass posterior. With the revised parallax from Gaia DR2, the total dynamical mass of HD 130948BC derived from astrometric analysis reported in Dupuy \& Liu (2017) of $M_{\text {dyn }}=115.4_{-2.1}^{+2.2} M_{\mathrm{J}}$ is updated to $M_{\text {dyn }}=116.2_{-0.8}^{+0.9} M_{\mathrm{J}}$. The model bolometric luminosity is calculated by log-linearly interpolating the evolutionary model grids. The log-likelihood is calculated from the residuals of the model luminosities and measured luminosities of HD 130948BC and their respective errors. Uniform bounded priors were used for the age of the system. The posterior distribution of the masses of each component and the age of the system was sampled using emcee. The credible regions from the resulting marginalized posteriors are reported for both sets of models in Table 4. The apparent bolometric fluxes from HD 130948B and C at best fit are $(1.31 \pm 0.03) \times 10^{-11} \mathrm{erg} \mathrm{s}^{-1} \mathrm{~cm}^{-2}$ and $(1.07 \pm 0.03) \times$ $10^{-11} \mathrm{erg} \mathrm{s}^{-1} \mathrm{~cm}^{-2}$, respectively.

\section{Discussion}

Independent validation of atmospheric and evolutionary models is critical for characterizing the atmospheres of directly imaged extrasolar planets and brown dwarfs. The masses of directly imaged exoplanets and brown dwarfs are generally poorly constrained owing to the difficulty in independently measuring masses, and inferences being extremely model dependent. However, mass is fundamentally important to test models of giant planet formation and empirically calibrate
Table 4

Evolutionary-model-inferred Properties of HD 130948BC

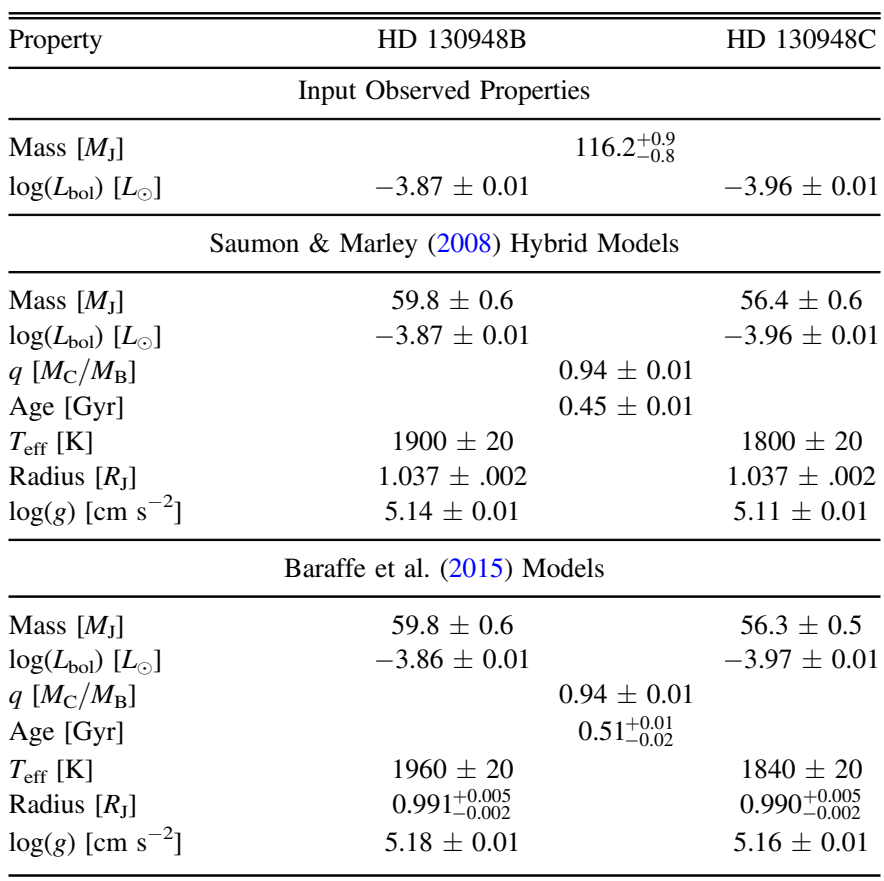

substellar evolutionary models. HD 130948B and C are some of the only brown dwarfs with independently measured mass, age, and luminosity to probe these models (Dupuy et al. 2009, 2014; Konopacky et al. 2010; Dupuy \& Liu 2017).

\subsection{Evolutionary and Atmospheric Models}

The atmosphere-model-fitting is independent of the evolutionary models (apart from physically motivated bounds on the radius prior). The evolutionary model fits are only informed by the modeled bolometric luminosities of HD 130948B and C, which are driven by the data, and independent dynamical mass measurements. The different models are not strictly expected to derive consistent atmosphere quantities. Specifically, nearinfrared spectroscopy has been shown to produce atmospheric model fits that are discrepant by $250 \mathrm{~K}$ from evolutionary model fits to the same data set (Dupuy et al. 2010).

Our fits to $L$-band spectra have similarly derived temperatures $\sim 250 \mathrm{~K}$ warmer than the other two methods (Tables 2 and 3). The coarse sampling of atmospheric parameters for the atmospheric model grid also limits what can be determined from the surface gravity measurement. At ALES spectral resolution and this temperature regime, the $L$-band does not vary significantly with surface gravity, and there are no prominent features that are highly gravity dependent. While the $L$-band is not particularly diagnostic of atmospheric parameters for hotter objects, the $L$-band becomes critical at lower temperatures (e.g., Skemer et al. 2014; Barman et al. 2015).

However, atmospheric model fitting to the combination of $J H K$ photometry and $L$-band spectra for HD 130948 B and C has resulted in posteriors that are completely consistent with evolutionary-model-derived quantities, illustrating the importance of extended wavelength coverage for substellar objects. Figure 7 depicts isomass lines and isochrones in effective temperature-surface gravity space with evolutionary models from Baraffe et al. (2015). The red and blue regions denote the 

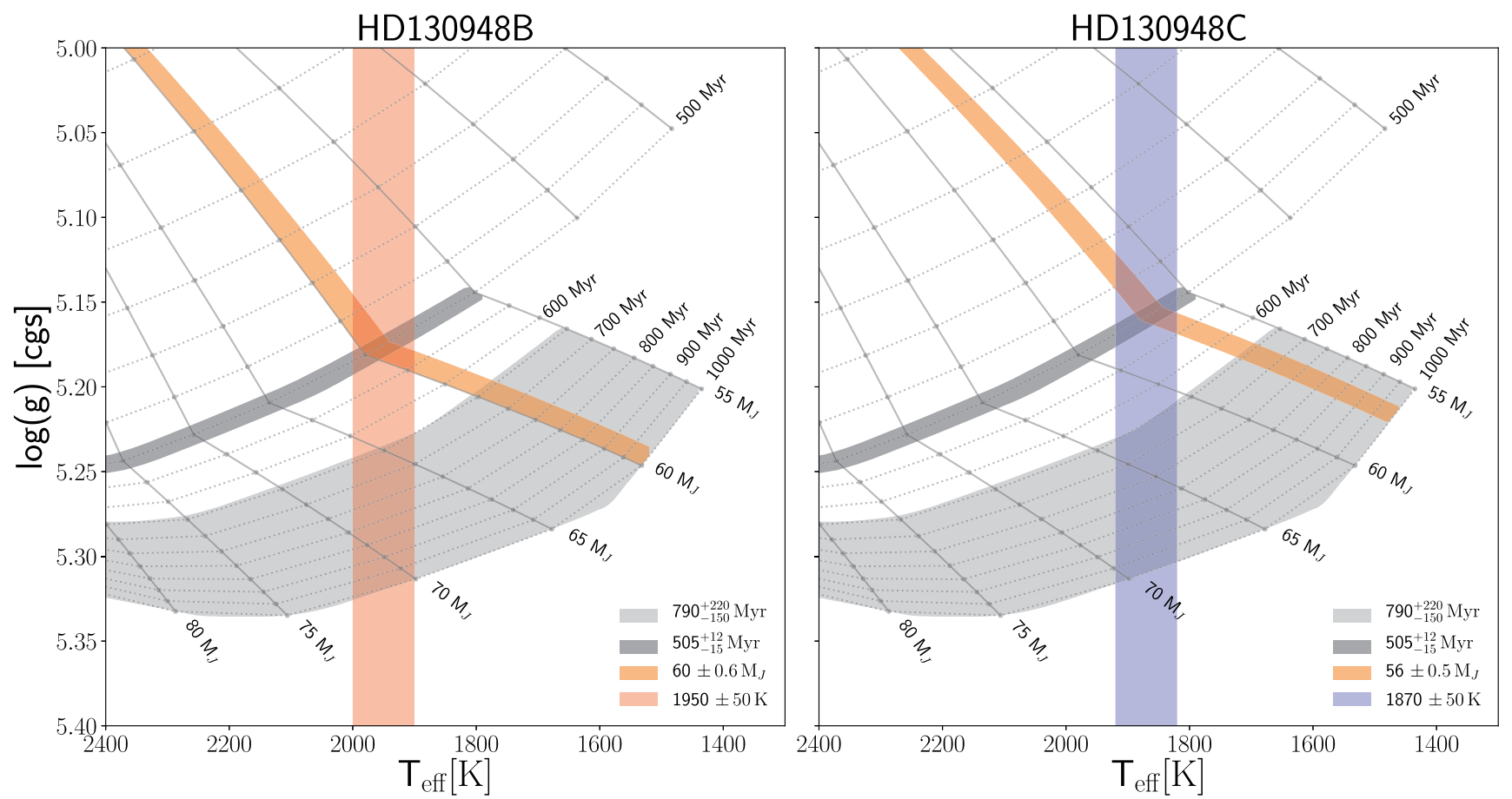

Figure 7. Isomass lines in solid black and isochrones in dashed black lines from Baraffe et al. (2015). The light gray region corresponds to the gyrochronology constraint from the primary star. The dark gray region corresponds to the $68 \%$ credible regions of age derived from the evolutionary model fitting of HD $130948 \mathrm{BC}$ according to Section 5.2. The orange regions correspond to the credible regions of mass derived from the evolutionary model fitting of HD 130948BC. The red and blue regions correspond to the credible regions of temperature derived from the atmospheric model fitting of HD 130948BC.

credible regions of effective temperature (and surface gravity) from atmosphere-model fitting, including the grid spacing errors set to half the grid spacings $\left(\sigma_{T_{\text {eff }}}=50 \mathrm{~K}, \sigma_{\log g}=0.25\right.$ dex). The publicly available evolutionary models did not continue past $1 \mathrm{Gyr}$ in this mass regime.

\subsection{Age of $H D 130948$}

We assume coevolution of the binary brown dwarfs with the primary star for the following discussion. With the method described in Section 5.2, the Baraffe et al. (2015, hereafter BHAC15) and hybrid Saumon \& Marley (2008, hereafter SM08) evolutionary models were used to derive the ages of $0.51_{-0.02}^{+0.01}$ Gyr and $0.45 \pm 0.01 \mathrm{Gyr}$ for HD 130948BC, respectively. Both evolutionary-model-derived ages are consistent with the age of HD 130948A of $0.5 \pm 0.3 \mathrm{Gyr}$ as traced by the Ca II HK emission (Dupuy et al. 2009), the previous evolutionary-model-derived age of $0.44 \pm 0.04$ (Dupuy \& Liu 2017), and the Barnes (2007) relationship for a gyrochronological age of $0.65_{-0.10}^{+0.13} \mathrm{Gyr}$.

The gyrochronology relation from Mamajek \& Hillenbrand (2008) that results in an age of $0.79_{-0.15}^{+0.22} \mathrm{Gyr}$ does remain an outstanding topic of discussion, due to its adoption as the age of the system in Dupuy et al. (2009).

One factor in this adoption is the observation that the $B-V$ color of HD 130948A suggests an age marginally consistent with, if not older than, the Hyades cluster. The Hyades cluster was believed to have a tight age constraint of $625 \pm 50 \mathrm{Myr}$ (Perryman et al. 1998). However, Brandt \& Huang (2015a, 2015b) have fit rotating stellar models to main-sequence turnoff Hyads to measure the age of the Hyades cluster to be older and with wider spread $(750 \pm 100 \mathrm{Myr})$. If the Hyades are systematically older, gyrochronology relations would need to be recalibrated to ameliorate the updated age (Douglas et al. 2016).
It should also be noted that Gossage et al. (2018) used a different prescription of rotating stellar models and derived an age $\sim 680 \mathrm{Myr}$, which is roughly consistent with the canonical age of the cluster. For HD 130948A to be strictly older than the Hyades, the bolometric luminosities of HD 130948B and C would be considerably overluminous compared to the predictions from evolutionary models.

Several possible explanations exist for the age discrepancy, including (1) the treatment of clouds, metallicities, and atmospheric opacities playing a major role evolutionary models (Burrows et al. 2011), (2) evolutionary models systematically overpredicting cooling rates of substellar objects (Dupuy et al. 2011), (3) very strong, interior magnetic fields inhibiting the onset of convection in HD 130948BC (Mullan \& MacDonald 2010), (4) the efficiency of convection that decreases for fast-rotating, highly magnetic low-mass stars extending to substellar evolution (Chabrier et al. 2007), (5) atypical stellar rotation which can be induced from formation via gravitational instability in a longlived, massive circumstellar disk (Dupuy et al. 2014), and (6) systematic offsets in gyrochronology relations for field $\mathrm{G}$ stars (Mamajek \& Hillenbrand 2008). Probing these explanations is beyond the scope of this paper, but will become critical to investigate cooler objects with any precision.

The luminosity evolution for HD 130948B and C is depicted in Figure 8. The individual lines and line width correspond to the median and $68 \%$ credible regions of the best-fit mass as derived in Section 5.2, propagated from $100 \mathrm{Myr}$ to $1 \mathrm{Gyr}$ using the two evolutionary models. This plot is qualitatively identical to Figure 10 of Dupuy et al. (2009).

The consistency of atmospheric quantities derived from evolutionary model fitting and atmospheric model fitting for HD 130948BC provides complementary evidence to support the age derived from the evolutionary model fits. We chose to 


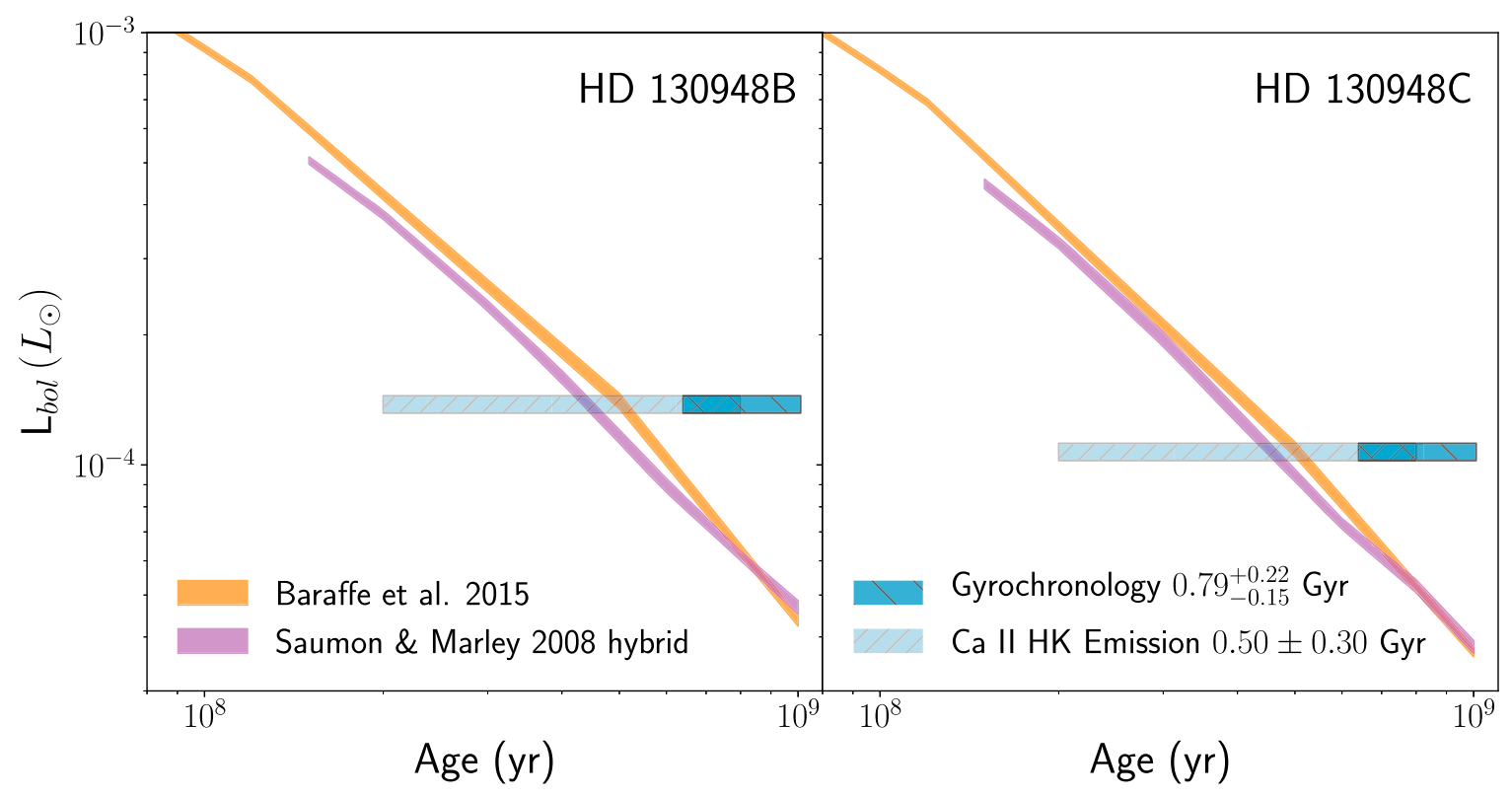

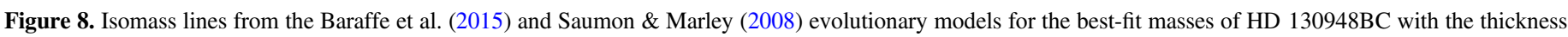

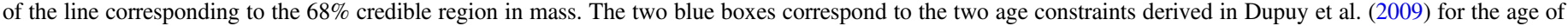
the primary HD 130948A through gyrochronology and chromospheric activity.

focus on the BHAC15 evolutionary models. A Monte Carlo simulation was performed using draws of the atmospheremodel-derived $(\mathrm{JHK}+\mathrm{L})$ effective temperature posteriors and the individual mass posteriors derived in the evolutionary model fitting, and propagated through the evolutionary model grid to obtain an average age for each draw of an effective temperature and mass for $\mathrm{B}$ and $\mathrm{C}$. The age derived using the BHAC15 models was $0.50 \pm 0.07 \mathrm{Gyr}$, which is consistent with the age traced by Ca II HK emission and the Barnes (2007) gyrochronology age.

The derived age of HD 130948BC is younger than age estimates of the Hyades, while the $B-V$ color and rotational period of HD 130948A lie in parameter space beyond the $625 \mathrm{Myr}$ isochrone of the Hyades (Perryman et al. 1997; Gaidos et al. 2000; Mamajek \& Hillenbrand 2008). Leveraging the consistency of the atmospheric and evolutionary models for HD 130948BC and assuming the binary and primary are coeval, we invoke anomalous stellar angular momentum loss as an explanation for the systematically older age estimates from gyrochronology relationships (Barnes 2007; Mamajek \& Hillenbrand 2008) compared to evolutionary-model-derived ages for HD 130948.

This explanation, however, is not sufficient to describe the behavior of other overluminous substellar objects, such as Gl 417BC. The Gl 417 system is a hierarchical triple system similar to HD 130948, with the Gl 417BC brown dwarf binary separated $90^{\prime \prime}$ from their primary star Gl 417A, and therefore ineffective at driving anomalous stellar angular momentum loss (Dupuy et al. 2014).

\subsection{Spectrophotometric Characterization of HD $130948 B C$}

Section 5.1 presents the fitting of the spectral energy density with three distinct methods, each of which produce broadly consistent atmosphere parameters for two L4 brown dwarfs. At around 1800-2000 K, the spectral features of brown dwarfs in the $L$-band are dominated by the $\mathrm{H}_{2} \mathrm{O}$ pseudo-continuum (Barman et al. 2015). At ALES resolution, cooler temperature brown dwarfs $(<1800 \mathrm{~K})$ begin to exhibit $\mathrm{CH}_{4} \mathrm{PQR}$ branch absorption that suppresses the water pseudo-continuum near $3.3 \mu \mathrm{m}$. No significant methane absorption is evident in the $L$-band spectra of HD 130948BC, placing the pair earlier than L5 spectral type. Due to the lack of spectroscopic standards in the $L$-band, we defer to the spectral-type determination of $\mathrm{L} 4 \pm 1$ from the literature (Goto et al. 2002).

The color-magnitude diagram with field brown dwarfs and HD 130948BC is plotted in Figure 9. Synthetic $L^{\prime} / W 1$ photometry was calculated by using the MKO $L^{\prime} / W I S E-W 1$ filter curve and the ALES spectra of HD 130948BC. Synthetic $L^{\prime}$ magnitudes of HD $130948 \mathrm{BC}$ are $11.308 \pm 0.034 \mathrm{mag}$ and $11.461 \pm 0.039 \mathrm{mag}$, respectively. The delta $L^{\prime}$ magnitude between HD 130948B and HD 130948C is $0.153 \pm 0.034$. The calculated $K-L^{\prime}$ values for HD 130948BC are $1.040 \pm 0.042$ mag and $1.080 \pm 0.042 \mathrm{mag}$, respectively. The synthetic $W 1$ magnitudes of HD 130948BC are $11.727 \pm 0.036 \mathrm{mag}$ and $11.856 \pm 0.043 \mathrm{mag}$, respectively. The delta $W 1$ magnitude between HD 130948B and HD $130948 \mathrm{C}$ is $0.129 \pm 0.037$. The calculated $K-W 1$ values for HD $130948 \mathrm{BC}$ are $0.622 \pm 0.042$ mag and $0.689 \pm 0.042 \mathrm{mag}$, respectively.

Near-infrared spectra of HD 130948BC exist, but the observation suffered from differential slit loss (Goto et al. 2002). The continuum contains the temperature and gravity information, so we chose not to include this data set for spectral fitting. However, Goto et al. did identify 2MASSW J00361617 +1821104 (L4) as being the best-matched template spectrum for both observed HD 130948BC medium-resolution $H K$ spectra. Photometry of 2MASSW J00361617+1821104 was used to calculated a $K-L^{\prime}$ of $0.96 \pm 0.058$ (Leggett et al. 2002; Knapp et al. 2004). Comparing the three L4 brown dwarfs, both HD 130948BC are slightly redder, perhaps due to adolescence.

There also exists resolved optical photometry of the binary from Hubble Space Telescope/ACS-HRC (Dupuy et al. 2009). These data comprise flux ratios of HD 130948BC in four red-optical bandpasses. In order to simultaneously fit 

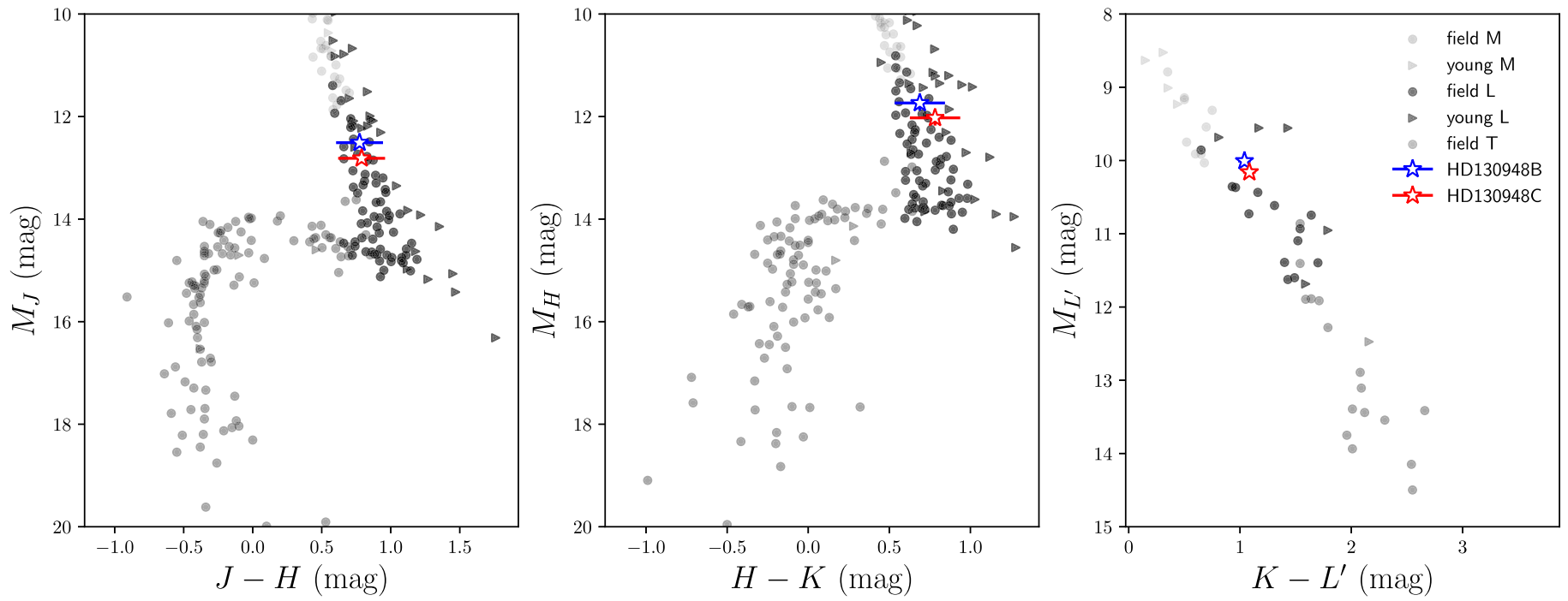

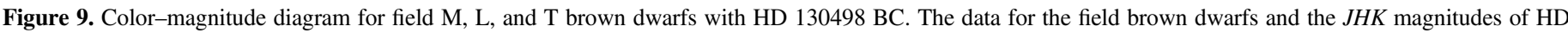
130948BC are from the Database of Ultracool Parallaxes (Dupuy \& Liu 2012; Dupuy \& Kraus 2013; Liu et al. 2016) and Dupuy \& Liu (2017), respectively.

red-optical photometry, NIR photometry, and $L$-band spectroscopy, our model grid would need to be expanded considerably to cover a much broader range of parameters (e.g., abundances, cloud properties, non-equilibrium chemistry). An extensive parameter search is beyond the intended scope of this paper. The synthetic flux ratios calculated for the $J H K+L$ best fit for $F 850 L P, F R 914 M(8626 \AA)$, $F R 914 M(9402 \AA)$, and $F R 914 M(10248 \AA)$ are $0.10 \pm 0.03$, $0.31 \pm 0.03,0.40 \pm 0.03$, and $0.44 \pm 0.02$, respectively.

\section{Conclusion}

We obtained 2.9-4.1 $\mu \mathrm{m}$ spectra of HD 130948BC with the ALES IFS. This is the first time an adaptive-optics-fed IFS has been used at these wavelengths. We demonstrated that atmospheric models are able to reproduce the SEDs of these benchmark brown dwarfs. The $J H K$ photometry and $L$-band spectra become potent constraints when used in tandem, recovering parameters consistent with the evolutionary model fits. Our results suggest that low-resolution $L$-band spectra can ameliorate the discrepancy between atmosphere fits and evolutionary model fits for objects with only $J H K$ photometry, making ALES a powerful tool in aiding our understanding of evolutionary and atmosphere models.

Our determination that the ALES spectra can aid nearinfrared measurements in characterizing the atmosphere of HD $130498 \mathrm{BC}$ has been the culmination of the development of a versatile pipeline and observation strategy that sets ALES as a new instrument capable of characterizing the thermal spectral properties of directly imaged planets and brown dwarfs. When the James Webb Space Telescope (JWST) launches, there will be enormous scientific opportunity for studying exoplanets in the thermal infrared. ALES will be complementary to JWST. While ALES is less sensitive, it probes smaller inner working angles, especially in the context of spectroscopy. Both JWST and ALES will increase the wavelength range over which we study directly imaged planets, which will be especially important as we begin to study colder exoplanets.
We would like to thank the anonymous referee for the constructive criticism that has greatly benefited the clarity of our manuscript.

The LBT is an international collaboration among institutions in the United States, Italy, and Germany. LBT Corporation partners are The University of Arizona on behalf of the Arizona university system; Istituto Nazionale di Astrofisica, Italy; LBT Beteiligungsgesellschaft, Germany, representing the MaxPlanck Society, the Astrophysical Institute Potsdam, and Heidelberg University; The Ohio State University, and The Research Corporation, on behalf of The University of Notre Dame, University of Minnesota, and University of Virginia. This paper is based on work funded by NSF Grants 1608834 and 1614320 .

J.M.S. is supported by NASA through Hubble Fellowship grant HST-HF2-51398.001-A awarded by the Space Telescope Science Institute, which is operated by the Association of Universities for Research in Astronomy, Inc., for NASA, under contract NAS4-26555. Barman is supported by NSF AAG awards 1405505 and 1614492, and NASA XRP award NNX17AB63G. Z.W.B. is supported by the National Science Foundation Graduate Research Fellowship under grant DGE 1842400.

Facility: LBT (LBTI/LMIRcam/ALES).

Software: Astropy (Astropy Collaboration et al. 2018), matplotlib (Hunter 2007), SciPy (Jones et al. 2001), NumPy (van der Walt et al. 2011), emcee (Foreman-Mackey et al. 2013), jupyter (http://jupyter.org/), SAOImage DS9.

\section{Appendix \\ Bayesian Parameter Estimation for PSF Fitting}

In the following procedure, the subscript $\lambda$ will be dropped for clarity; the procedure implicitly applies to each wavelength slice in the data cube. For each wavelength slice of the PSF and binary, there are six parameters $\phi$ defining a model of the two brown dwarfs with two shifted PSFs: the position $\left(y_{\mathrm{B}}, x_{\mathrm{B}}\right)$ of HD 130948B, the position angle $\theta$ and the projected separation of the brown dwarfs $\rho$, the contrast ratios $\alpha_{\mathrm{B}}$ and $\alpha_{\mathrm{C}}$ of HD 130948BC with respect to the PSF of HD 130948A, and a hyperparameter $\ell$ corresponding to a spatial correlation length. 
An additive factor to quantify sky background offsets was determined empirically to be consistent with zero, and therefore not included.

The binary $\mathcal{D}$ is modeled by shifting and scaling the PSF, $\mathcal{A}$, to each object. The unscaled model for HD 130948B is $\mathcal{A}_{\mathrm{B}}(\phi)=\mathcal{A}\left[y_{\mathrm{B}}, x_{\mathrm{B}}\right]$ and the unscaled model for HD $130948 \mathrm{C}$ is $\mathcal{A}_{\mathrm{C}}(\phi)=\mathcal{A}\left[y_{\mathrm{B}}+\rho \sin \theta, x_{\mathrm{B}}+\rho \cos \theta\right]$, where $[\cdot, \cdot]$ denotes the translation function of an image. The binary was coarsely centered on a $17 \times 17$ pixel fitting region that defines the reference origin. The PSF was centered on a square-fitting region of area that was 10 pixels larger on all sides to ensure that any plausible shift of the PSF would populate the entire fitting region with data. The residuals between the data and the model are $R \equiv \mathcal{D}-\alpha_{\mathrm{B}} \mathcal{A}_{\mathrm{B}}(\phi)-\alpha_{\mathrm{C}} \mathcal{A}_{\mathrm{C}}(\phi)$.

The variance images of $\mathcal{A}_{\mathrm{B}}, \mathcal{A}_{\mathrm{C}}$, and $\mathcal{D}$ are propagated similarly, and are denoted $\sigma_{\mathrm{B}}^{2}, \sigma_{\mathrm{C}}^{2}$, and $\sigma_{\mathcal{D}}^{2}$. The uncorrelated uncertainty vector is $\sigma^{2}(\phi)=\sigma_{\mathcal{D}}^{2}+\alpha_{\mathrm{B}}^{2} \sigma_{\mathrm{B}}^{2}+\alpha_{\mathrm{C}}^{2} \sigma_{\mathrm{C}}^{2}$, which is converted into the covariance matrix $C_{\ell}$ using Equation (1). In principle, variance is not linear, and therefore, $\sigma_{\mathrm{B}}^{2}$ and $\sigma_{\mathrm{C}}^{2}$ cannot be interpolated simply by this translation function. However, the contribution of the PSF variance scaled by the square of the flux ratio already acts as a small perturbation of the variance image of the binary data. At the measured spatial correlation lengths, $\ell$, the contribution of the covariance terms is even higher order and therefore ignored. That being said, $C_{\ell}$ is still highly nonlinear in $\phi$, and therefore, this problem is not ideal for generalized least-squares estimators.

Bayes' theorem is used to write the posterior probability for $\phi$ and $\ell$ given $\mathcal{D}$ as $\mathcal{P}(\phi, \ell \mid \mathcal{D}) \propto \mathcal{L}(\mathcal{D} \mid \phi, \ell) \mathcal{P}(\phi, \ell)$. To quantify the probability of the data conditioned on the model, we adopted a multivariate Gaussian likelihood function

$$
-2 \ln \mathcal{L}(\mathcal{D} \mid \phi, \ell)=R^{T} C_{\ell}^{-1} R+\ln \left|C_{\ell}\right|+N_{\text {pix }} \ln 2 \pi .
$$

We employ a uniform, bounded prior $\mathcal{P}(\phi, \ell)$. The bounds exclude PSF shifts off the fitting region, negative separations $\rho$ of the PSFs or $\theta \pm \pi$ (corresponding to $y_{\mathrm{B}}, x_{\mathrm{B}}$ instead describing the position of HD 130948C), and extremely large or small $\alpha_{\mathrm{B}}$ and $\alpha_{\mathrm{C}}$. Spatial correlation lengths are positivedefinite and bounded above by the size of the fitting region.

We used emcee to sample the posterior distribution for each wavelength slice independently. We initialized 100 walkers with a guess $\phi$ vector plus Gaussian noise at an amplitude of $\phi \times 10^{-4}$. The MCMC sampler was run for 1000 steps after 1000 burn-in steps for each wavelength. The matrix inversion, multiplication, and determinant of the Hermitian, positivedefinite covariance matrix was calculated using the Cholesky decomposition to take advantage of numerical stability.

The resulting posterior distributions at each wavelength were marginalized over position, contrast, and spatial correlation length terms, and combined jointly to calculate the median values for position angle and separation across all wavelengths, denoted $\theta^{*}$ and $\rho^{*}$. The plate-scale error of $0.1 \mathrm{mas} /$ spaxel was propagated with Monte Carlo when converting the separation in spaxels to angular separation in milliarcseconds. The standard deviations $\sigma_{\theta^{*}}$ and $\sigma_{\rho}{ }^{*}$ were calculated from the joint distribution assuming critical Nyquist sampling. This assumption is supported in Section 5.1.1. The values adopted for position angle and separation are $\theta^{*}=137^{\circ} .0 \pm 0.2$ and $\rho^{*}=107.8 \pm 0.3$ mas, respectively.

The second step uses the same uniform, bounded priors $\mathcal{P}(\phi)$ from the first step, except for position angle and separation. The priors for position angle and separation are updated to be
$\mathcal{P}(\theta)=\mathcal{N}\left[\theta ; \theta^{*}, \sigma_{\theta^{*}}^{2}\right]$ and $\mathcal{P}(\rho)=\mathcal{N}\left[\rho ; \rho^{*}, \sigma_{\rho^{*}}^{2}\right]$. The posterior distribution was sampled again for each wavelength under the same conditions stated before.

The correlations evident in Figure 4 manifest as a weak degeneracy between the position of the brown dwarfs and flux ratios with respect to the PSF: the model binary built from two shifted PSFs will "exchange" flux when they are mislocated. A precision astrometric solution or $L$-band flux ratios of the brown dwarfs would be necessary to break this degeneracy (with more assumptions). Without such information, we do not probe this degeneracy further.

\section{ORCID iDs}

Zackery W. Briesemeister (i) https://orcid.org/0000-00021764-2494

Andrew J. Skemer (i) https://orcid.org/0000-0001-6098-3924

Jordan M. Stone (iD https://orcid.org/0000-0003-0454-3718

Travis S. Barman (10 https://orcid.org/0000-0002-7129-3002

Jarron Leisenring (iD https://orcid.org/0000-0002-0834-6140

Eckhart Spalding (i) https://orcid.org/0000-0003-3819-0076

\section{References}

Astropy Collaboration, Price-Whelan, A. M., Sipőcz, B. M., et al. 2018, AJ, 156,123

Bailey, V. P., Hinz, P. M., Puglisi, A. T., et al. 2014, Proc. SPIE, 9148, 914803 Baraffe, I., Homeier, D., Allard, F., \& Chabrier, G. 2015, A\&A, 577, A42

Barman, T. S., Konopacky, Q. M., Macintosh, B., \& Marois, C. 2015, ApJ, 804,61

Barman, T. S., Macintosh, B., Konopacky, Q. M., \& Marois, C. 2011, ApJ, 733, 65

Barnes, S. A. 2007, ApJ, 669, 1167

Beletic, J. W., Blank, R., Gulbransen, D., et al. 2008, Proc. SPIE, 7021 , $70210 \mathrm{H}$

Bjoraker, G. L., Larson, H. P., \& Kunde, V. G. 1986, Icar, 66, 579

Bowler, B. P., Liu, M. C., Dupuy, T. J., \& Cushing, M. C. 2010, ApJ, 723, 850

Brandt, T. D., \& Huang, C. X. 2015a, ApJ, 807, 58

Brandt, T. D., \& Huang, C. X. 2015b, ApJ, 807, 24

Briesemeister, Z., Skemer, A. J., Stone, J. M., et al. 2018, Proc. SPIE, 10702, 107022Q

Brooks, S. P., \& Gelman, A. 1998, J. Comput. Graph. Stat., 7, 434

Burrows, A., Heng, K., \& Nampaisarn, T. 2011, ApJ, 736, 47

Burrows, A., \& Liebert, J. 1993, RvMP, 65, 301

Burrows, A., Marley, M., Hubbard, W. B., et al. 1997, ApJ, 491, 856

Chabrier, G., Gallardo, J., \& Baraffe, I. 2007, A\&A, 472, L17

Claudi, R. U., Turatto, M., Gratton, R. G., et al. 2008, Proc. SPIE, 7014, 70143E

Crossfield, I. J. M. 2014, A\&A, 566, A130

Currie, T., Burrows, A., Itoh, Y., et al. 2011, ApJ, 729, 128

Cushing, M. C., Marley, M. S., Saumon, D., et al. 2008, ApJ, 678, 1372

Czekala, I., Andrews, S. M., Mandel, K. S., Hogg, D. W., \& Green, G. M. 2015, ApJ, 812, 128

Douglas, S. T., Agüeros, M. A., Covey, K. R., et al. 2016, ApJ, 822, 47

Dupuy, T. J., \& Kraus, A. L. 2013, Sci, 341, 1492

Dupuy, T. J., \& Liu, M. C. 2012, ApJS, 201, 19

Dupuy, T. J., \& Liu, M. C. 2017, ApJS, 231, 15

Dupuy, T. J., Liu, M. C., Bowler, B. P., et al. 2010, ApJ, 721, 1725

Dupuy, T. J., Liu, M. C., \& Ireland, M. J. 2009, ApJ, 692, 729

Dupuy, T. J., Liu, M. C., \& Ireland, M. J. 2011, in ASP Conf. Ser. 448, 16th Cambridge Workshop on Cool Stars, Stellar Systems, and the Sun, ed. C. Johns-Krull, M. K. Browning, \& A. A. West (San Francisco, CA: ASP), 111

Dupuy, T. J., Liu, M. C., \& Ireland, M. J. 2014, ApJ, 790, 133

Eiroa, C., Marshall, J. P., Mora, A., et al. 2013, A\&A, 555, A11

Esposito, S., Riccardi, A., Pinna, E., et al. 2011, Proc. SPIE, 8149, 814902

Foreman-Mackey, D., Hogg, D. W., Lang, D., \& Goodman, J. 2013, PASP, 125,306

Gaia Collaboration, Brown, A. G. A., Vallenari, A., et al. 2016, A\&A, 595, A2 Gaia Collaboration, Brown, A. G. A., Vallenari, A., et al. 2018, A\&A, 616, A1 Gaidos, E. J., Henry, G. W., \& Henry, S. M. 2000, AJ, 120, 1006

Gelman, A., \& Rubin, D. B. 1992, StaSc, 7, 457 
Ginski, C., Neuhäuser, R., Mugrauer, M., Schmidt, T. O. B., \& Adam, C. 2013, MNRAS, 434, 671

Goodman, J., \& Weare, J. 2010, CAMCOS, 5, 65

Gossage, S., Conroy, C., Dotter, A., et al. 2018, ApJ, 863, 67

Goto, M., Kobayashi, N., Terada, H., et al. 2002, ApJL, 567, L59

Greco, J. P., \& Brandt, T. D. 2016, ApJ, 833, 134

Guizar-Sicairos, M., Thurman, S. T., \& Fienup, J. R. 2008, OptL, 33, 156

Hauschildt, P. H., Allard, F., \& Baron, E. 1999, ApJ, 512, 377

Hinkley, S., Oppenheimer, B. R., Zimmerman, N., et al. 2011, PASP, 123, 74

Hinz, P., Arbo, P., Bailey, V., et al. 2012, Proc. SPIE, 8445, 84450U

Hinz, P., Bailey, V. P., Defrère, D., et al. 2014, Proc. SPIE, 9146, 91460T

Hinz, P. M., Bippert-Plymate, T., Breuninger, A., et al. 2008a, Proc. SPIE, 7013, 701328

Hinz, P. M., Skemer, A., Stone, J., Montoya, O. M., \& Durney, O. 2018, Proc. SPIE, 10702, 107023L

Hinz, P. M., Solheid, E., Durney, O., \& Hoffmann, W. F. 2008b, Proc. SPIE, 7013, 701339

Horne, K. 1986, PASP, 98, 609

Hunter, J. D. 2007, CSE, 9, 90

Ingraham, P., Marley, M. S., Saumon, D., et al. 2014, ApJL, 794, L15

Jones, E., Oliphant, T., \& Peterson, P. 2001, SciPy: Open Source Scientific Tools for Python, http://www.scipy.org/

Knapp, G. R., Leggett, S. K., Fan, X., et al. 2004, AJ, 127, 3553

Konopacky, Q. M., Ghez, A. M., Barman, T. S., et al. 2010, ApJ, 711, 1087

Larkin, J., Barczys, M., Krabbe, A., et al. 2006, Proc. SPIE, 6269, 62691A

Leggett, S. K., Golimowski, D. A., Fan, X., et al. 2002, ApJ, 564, 452

Leisenring, J. M., Nelson, M. J., Hoffmann, W. F., et al. 2010, Proc. SPIE, $7735,77352 \mathrm{~N}$

Leisenring, J. M., Skrutskie, M. F., Hinz, P. M., et al. 2012, Proc. SPIE, 8446, $84464 \mathrm{~F}$

Liu, M. C., Dupuy, T. J., \& Allers, K. N. 2016, ApJ, 833, 96
Low, F. J., \& Davidson, A. W. 1969, BAAS, 1, 200

Macintosh, B. A., Graham, J. R., Palmer, D. W., et al. 2008, Proc. SPIE, 7015, 701518

Madhusudhan, N., Burrows, A., \& Currie, T. 2011, ApJ, 737, 34

Mamajek, E. E., \& Hillenbrand, L. A. 2008, ApJ, 687, 1264

Marley, M. S., Saumon, D., Cushing, M., et al. 2012, ApJ, 754, 135

McElwain, M. W., Brandt, T. D., Janson, M., et al. 2012, Proc. SPIE, 8446, 84469C

Morley, C. V., Fortney, J. J., Marley, M. S., et al. 2012, ApJ, 756, 172

Morley, C. V., Marley, M. S., Fortney, J. J., et al. 2014, ApJ, 787, 78

Morzinski, K. M., Males, J. R., Skemer, A. J., et al. 2015, ApJ, 815, 108

Mullan, D. J., \& MacDonald, J. 2010, ApJ, 713, 1249

Perryman, M. A. C., Brown, A. G. A., Lebreton, Y., et al. 1998, A\&A, 331, 81

Perryman, M. A. C., Lindegren, L., Kovalevsky, J., et al. 1997, A\&A, 323, L49

Potter, D., Martín, E. L., Cushing, M. C., et al. 2002, ApJL, 567, L133

Rajan, A., Rameau, J., De Rosa, R. J., et al. 2017, AJ, 154, 10

Saumon, D., \& Marley, M. S. 2008, ApJ, 689, 1327

Skemer, A. J., Hinz, P., Montoya, M., et al. 2015, Proc. SPIE, 9605, 96051D

Skemer, A. J., Hinz, P., Stone, J., et al. 2018, Proc. SPIE, 10702, 107020C

Skemer, A. J., Hinz, P. M., Esposito, S., et al. 2012, ApJ, 753, 14

Skemer, A. J., Marley, M. S., Hinz, P. M., et al. 2014, ApJ, 792, 17

Skrutskie, M. F., Jones, T., Hinz, P., et al. 2010, Proc. SPIE, 7735, 77353H

Stephens, D. C., Leggett, S. K., Cushing, M. C., et al. 2009, ApJ, 702, 154 Stevenson, D. J. 1991, ARA\&A, 29, 163

Stone, J. M., Skemer, A. J., Hinz, P., et al. 2018, Proc. SPIE, 10702, 107023F

Tokunaga, A. T., Simons, D. A., \& Vacca, W. D. 2002, PASP, 114, 180

Valenti, J. A., \& Fischer, D. A. 2005, ApJS, 159, 141

van der Walt, S., Colbert, S. C., \& Varoquaux, G. 2011, CSE, 13, 22

Wang, J. J., Graham, J. R., Pueyo, L., et al. 2016, AJ, 152, 97

Wright, E. L., Eisenhardt, P. R. M., Mainzer, A. K., et al. 2010, AJ, 140, 1868

Zurlo, A., Vigan, A., Mesa, D., et al. 2014, A\&A, 572, A85 